\title{
Time to read semantically related sentences*
}

\author{
JAMES G. GREENO and DAVID L. NOREEN \\ University of Michigan, Ann Arbor, Michigan 48104
}

\begin{abstract}
Ss read sentences specifying relations among concepts, with the sentences related to each other in different hierarchical structures. In the case where sentences had implicative relationships, time to read sentences low in the hierarchy was less if sentences higher in the hierarchy had been read previously, and sentences inconsistent with expectations based on earlier items took longer. However, when structural relations did not involve implication about content, time to read the sentences was not very different regardless of whether related sentences had been read previously.
\end{abstract}

Kintsch and Keenan (1972) have shown that the time to read a sentence is approximately linear with the amount of semantic content of the sentence, as measured by the number of propositions expressed by the sentence. There is considerable evidence that, when $S$ reads several sentences, the cognitive outcome of the process is a structure of semantically related information, representing the concepts and relations in the material read (Bransford \& Franks, 1971; Kintsch \& Monk, 1972; Potts, 1972; Sachs, 1967). It might be expected that, if a sentence $q$ is consistent with expectations $\mathbf{S}$ would develop on the basis of earlier sentences, then $q$ would be easier to assimilate than if $q$ was unrelated to earlier material; difficulty in processing would result if $q$ was inconsistent with expectations developed by reading earlier sentences. This idea was tested in the present experiment.

\section{METHOD}

Eight paid Ss studied 11 sets of seven sentences each, with sentences presented one at a time. The $S$ was told that each set of seven sentences formed a paragraph. The task was to read each sentence as quickly as possible and store its meaning in memory. Each sentence was typed on a card. The $E$ gave $S$ a card and simultaneously started a timer using a foot switch. When $S$ finished reading the sentence, $S$ turned the card over, whereupon $E$ stopped the timer. The $S$ then counted backward by twos, starting with a three-digit number on the back of the card. Counting was paced by a metronome that clicked once per second, starting when $\mathbf{E}$ stopped the timer. After about $15 \mathrm{sec}$ of counting. E stopped $S$ and $S$ was asked to recall the substance of the sentence.

After each set of seven sentences, a series of true-false items was presented. The $S$ was instructed to answer true if the test item was presented in the paragraph or if its truth could be inferred from sentences in the paragraph. The correct answer was false if the item was inconsistent with sentences in the paragraph or if the paragraph did not contain sufficient information to enable $S$ to decide that the item was true.

\section{Materials}

Three kinds of paragraphs were constructed, each containing seven sentences with information arranged in an hierarchical fashion. The form of the hierarchies is shown in $\mathrm{fig}$. 1 . One kind

*This study was supported by the National Science Foundation under Grant GB-31045. of paragraph gave a series of categorical (subset-superset) relationships. There were seven concepts: denote $a, b_{1}, b_{2}, c_{1}$, $c_{2}, c_{3}, c_{4}$. Seven sentences were given, with the same relationships presented in two different orders: bottom up $-c_{1} \subset b_{1}, c_{2} \subset b_{1}, a \supset b_{1}, a \supset c_{2}, c_{3} \subset b_{2}, c_{4} \subset b_{2}, a \supset b_{2}$; top down-a $\supset b_{1}, c_{1} \subset b_{1}, c_{2} \subset b_{1}, a \supset c_{2}, a \supset b_{2}, c_{3} \subset b_{2}$. $c_{4} \subset b_{2}$. An example of seven sentences actually used is the following. Peace-loving people include the farmers of Central Ugala $\left(a \supset b_{1}\right)$. The river people of Central Ugala are farmers $\left(c_{1} \subset b_{1}\right)$. The hill people of this region are farmers $\left(c_{2} \subset b_{1}\right)$. Peace-loving people include the hill people of Central Ugala $\left(a \supset c_{2}\right.$ ). Peace-loving people include the herders of this region $\left(a \supset b_{2}\right)$. The Fundalas of Central Ugala are herders $\left(c_{3} \subset b_{2}\right)$. Outcasts from other tribes of this region are herders $\left(c_{4} \subset b_{2}\right)$.

Other paragraphs presenting categorical information dealt with two types of planets in "blue" galaxies and two groups of cars, allegedly reviewed in recent issues of two auto magazines.

A second kind of paragraph presented ordering relations between concepts. An example of these was a paragraph about use of phonograph records, with one order of the sentences being the following: In a recent survey made by a library, the folk records were found to be used less of ten than the country and western records $\left(c_{1}<b_{1}\right)$. The blues records were found to be used less often than the country and western records $\left(c_{2}<b_{1}\right)$. In the same survey, the jazz records were found to be used more often than the country and western records $\left(a>b_{1}\right)$. The jazz records were found to be used more often than the blues records $\left(a>c_{2}\right)$. The children's records were found to be

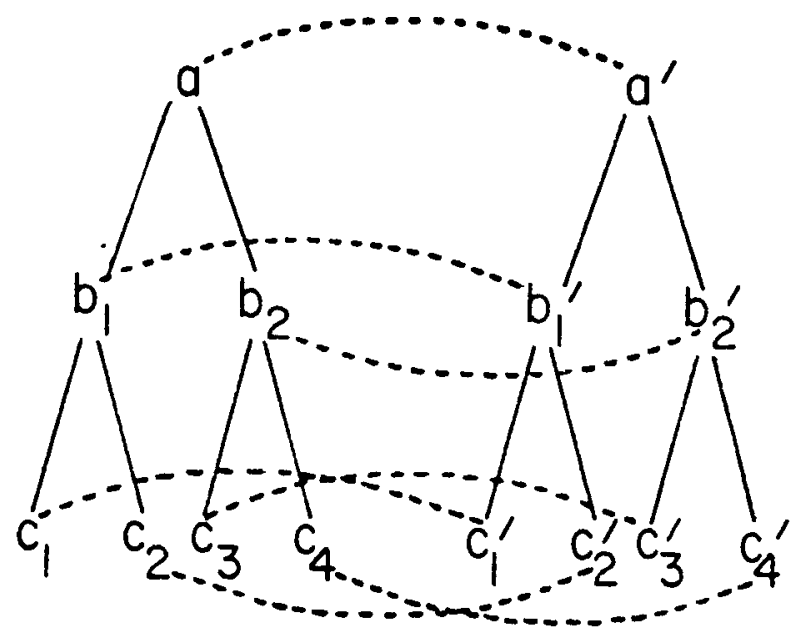

Fig. 1. Relations described in sentences. Categorical and ordering paragraphs gave relations between concepts in a single hierarchy. Comparison paragraphs gave relations between concepts at corresponding nodes of different hierarchies. 
Table 1

Mean Times to Read Sentences Comparing Concepts in Separate Hierarchies

\begin{tabular}{lccccrrr}
\hline \multicolumn{1}{c}{ Condition } & $\mathrm{a}$ & $\mathrm{b}_{1}$ & $\mathrm{~b}_{2}$ & $\mathrm{c}_{1}$ & $\mathrm{c}_{2}$ & \multicolumn{1}{c}{$\mathrm{c}_{3}$} \\
\hline Top Down, No Exception & 6.4 & 8.4 & 6.6 & 8.1 & 8.4 & 9.3 \\
Bottom Up, No Exception & 5.5 & 8.1 & 6.9 & 11.3 & 11.6 & 12.7 \\
Top Down, Exception at $c_{1}$ & 8.7 & 6.9 & 7.8 & 11.9 & 11.2 & 8.3 \\
Bottom Up, Exception at $c_{1}$ & 5.5 & 12.9 & 7.8 & 12.2 & 14.9 & 11.7 & 10.7 \\
\hline
\end{tabular}

used less often than the classical records $\left(c_{3}<b_{2}\right)$. The rock and roll records were found to be used less often than the classical records $\left(c_{4}<b_{2}\right)$. The jazz records were found to be used less often than the classical records $\left(a>b_{2}\right)$.

Other ordering relations were given in paragraphs dealing with several kinds of furniture in a store (dressers, tables, etc.). Bottom-up and top-down sequences were used, as with the categorical paragraphs.

All the paragraphs giving orderings and categorical relations gave the relations in the forms $a>b_{i}$ and $c_{j}<b_{i}$. This is apparently easier for $S s$ to assimilate than other forms (Huttenlocher, 1968).

The third kind of paragraph also gave hierarchically arranged information, but of a somewhat more complicated kind. Consider two sets of concepts, $S=a, b_{1}, b_{2}, c_{1}, c_{2}, c_{3}, c_{4}$ and $S^{\prime}=a^{\prime}, b_{1}{ }^{\prime}, b_{2}{ }^{\prime}, c_{1}{ }^{\prime}, c_{2}{ }^{\prime}, c_{3}{ }^{\prime}, c_{4}{ }^{\prime}$. The concepts in each set are related by subset-superset relations already in Ss' semantic memory structures. An ordering relation was applied between corresponding concepts in the two hierarchies. Formally, the relations were of the form $a>a^{\prime}, b_{1}>b_{1}{ }^{\prime}$, and so on. The sentences expressed these relations used in terms like "usually" or "most ___, making it possible to include exceptions to the general trend of the ordering given. The sequences in which sentences were presented were as follows: Bottom up $-c_{1}>c_{1}{ }^{\prime}\left(^{*}\right), \quad c_{2}>c_{2}{ }^{\prime}, \quad b_{1}>b_{1}{ }^{\prime}, \quad c_{3}>c_{3}{ }^{\prime}, \quad c_{4}>c_{4}{ }^{\prime}$, $\mathrm{b}_{2}>\mathrm{b}_{2}{ }^{\prime}$, a $>\mathrm{a}^{\prime}$. Top down $\left.-\mathrm{a}>\mathrm{a}^{\prime}, \quad \mathrm{b}_{1}>\mathrm{b}_{1}{ }^{\prime}, \mathrm{c}_{1}>\mathrm{c}_{1}{ }^{\prime}{ }^{*}\right)$, $c_{2}>c_{2}{ }^{\prime}, \quad b_{2}>b_{2}{ }^{\prime}, \quad c_{3}>c_{3}{ }^{\prime}, c_{4}>c_{4}{ }^{\prime}$. There were four paragraphs presenting these ordered relations between subclass hierarchies. In one paragraph from each sequence, the $c_{1}, c_{1}{ }^{\prime}$ relation was opposite to that mentioned above, thus constituting an exception to the general trend expressed in the other sentences. An example of sentences used is the following: The tall buildings in Midwest City usually seem to be built less soundly than the shorter buildings $\left(a^{\prime}<a\right)$. The office buildings with a small number of stories are generally more well built than those with a large number of stories $\left(b_{1}>b_{1}{ }^{\prime}\right)$. A department store in Midwest City that built a tall building used more expensive construction materials than another store that built a shorter building $\left(c_{1}{ }^{\prime}>c_{1}\right.$-exception). The towering structure of the Midwest Bank rests upon a shaky foundation, but the shorter National Bank of Midwest City was built exceptionally well $\left(c_{2}{ }^{\prime}<c_{2}\right)$. The high-rise apartment buildings are usually less sturdy than the shorter buildings $\left(b_{2}{ }^{\prime}<b_{2}\right)$. A recent small-scale low-cost public housing development was very well constructed, although an earlier high-rise public housing development had been built hastily and poorly $\left(c_{3}>c_{3}{ }^{\prime}\right)$. A private realty firm has a number of soundly built short buildings, but their tall buildings appear to be built carelessly $\left(c_{4}>c_{4}{ }^{\prime}\right)$.

Other paragraphs giving comparative information involving two hierarchical sets of concepts were about the priorities given to air and naval armaments in the Slobbovian Department of Defense, the relative amounts of interest that George has in literature and music, the relative importance of cleverness and true skill in achieving status in the island kingdom of Guelka, and the degree to which Hector likes older and younger people in his school and family.

Three paragraphs were given as practice; these were the paragraph comparing skill and cleverness among Guelkans, the paragraph constructing a categorical hierarchy about planets, and the ordering hierarchy about sales of furniture. The remaining eight paragraphs were presented in four different sequences, each sequence involving a different assignment of paragraphs to top-down and bottom-up orders and with or without exceptions in the comparative paragraphs. Two Ss were tested in each of the four sequences. The average results in each condition include an equal number of uses of each specific paragraph used in the experiment.

The true-false items given at the end of each paragraph were intended mainly to motivate $S$ to integrate the material presented in the individual sentences. For the comparative paragraphs, $\mathrm{S}$ was shown either a sentence that had been shown or the contradiction of a sentence, with a single item corresponding to each sentence shown in the paragraph. These items were shown in the same order for all paragraphs, the order being $a, b_{1}, b_{2}, c_{1}, c_{2}, c_{3}, c_{4}$. For the orderings and categorical paragraphs a variety of relations were tested, with a single set of eight items constructed for each paragraph and given in an order that did not vary from $S$ to $S$, though the order in which relations were tested varied between paragraphs.

\section{Results}

A high percentage of sentences were paraphrased correctly after backward counting. In 19 out of 448 cases, the content was incorrectly stated after the 15 -sec immediate retention interval.

Average times to read the sentences are given in Tables 1 and 2. Regarding the reliability of these numbers, it is probably best to consider that both variation among $\mathrm{Ss}$ and variation among different sentences were random sources of variation. On this interpretation, the estimated random variance within conditions was 27.01 , and this corresponds to a standard error of each mean equal to $1.84 \mathrm{sec}$. It is of interest that a considerable portion of this variance was produced by differences among Ss; separating variance due to $\mathrm{S}$ differences as a random factor gives an estimated variance component of 15.37 , leaving variance within conditions for individual Ss of 11.64. A test of the effect of difference between materials used in the same condition was carried out; there was no evidence that the different assignments of sentences to conditions contributed any significant amount of variance $(\mathrm{F}<1.0)$.

Results of greatest interest were obtained with the paragraphs in which two categorical hierarchies were compared. Average times taken to read these sentences are in Table 1. There are two facts of interest. First, there is a substantial effect on the time taken to read low sentences in the hierarchy, depending on whether sentences from higher levels have preceded them. In the paragraphs having no exceptions, the four sentences at the lowest level were read in 3.7 fewer seconds when the sequence of sentences was top down than when it was 
bottom up. The statistical comparison carried out is the interaction between the level of the sentence in the hierarchy and the order of presentation; that is, $(\bar{c}-a)_{B}-(\bar{c}-a)_{T}$, where $\bar{c}$ is the average time for all the c-level sentences, $a$ is the time for the a-level sentences, and subscripts $\mathrm{B}$ and $\mathrm{T}$ refer to bottom-up and top-down sequences. For the comparison paragraphs without exception, the $90 \%$ confidence interval for this contrast was $4.45 \pm 2.33 \mathrm{sec}$. (For 95\%, $\mathrm{Cl}=4.45 \pm 2.91$.) For this and other interval estimates of contrasts, the calculation was carried out using each S's score for the contrast and computing a simple $t$ statistic for the eight Ss.

The second interesting fact involves the time taken to read a sentence that is not consistent with expectations based on sentences read earlier. In the top-down condition, when a sentence at Node $c_{1}$ was inconsistent with earlier sentences it took longer than when it was consistent, as did the following sentence at Node $c_{2}$. In the bottom-up condition, the sentence at Node $c_{1}$ was the first sentence read. The two sentences that followed-at Nodes $c_{2}$ and $b_{1}$-took longer than they did in the bottom-up condition where all sentences were semantically consistent. The $90 \%$ confidence interval for the average of these effects was $3.01 \pm 3.01 \mathrm{sec}$. (For $95 \%, \mathrm{CI}=3.01 \pm 3.76$.)

The average reading times for sentences in the categorical and ordering hierarchies are given in Table 2. The interesting fact here involves the time taken to add relations to the network that relate to already built nodes compared to assimilating those same propositions before the related information was encountered. The strongest comparison involves all four of the lowest level sentences vs the two highest level sentences in the hierarchy. Comparisons were made between the top-down condition, where the lowest level sentences were given after the higher level sentences, and the bottom-up condition, where the reverse was true. In the categorical hierarchies, the interaction between level and sequence had a $90 \%$ confidence interval: $0.78 \pm 1.16 \mathrm{sec}$. For the hierarchies of ordering relations, the $90 \%$ confidence interval was $-1.42 \pm 1.90$.

The true-false items given at the end of each paragraph yielded only one finding of interest. There were so few errors in the questions dealing with categorical hierarchies $[(\mathrm{p}(\mathrm{err})=.08]$ and comparisons $[p(e r r)=.05]$ that we did not carry out an analysis of which kinds of questions produced the errors. In the hierarchies of orderings, most of the errors were false positives and occurred on items $b_{1}>c_{3}, c_{2}>c_{3}$, $b_{1}>b_{2}$ [overall $p(e r r)=.56$ ]. For the items with "true" as the correct response, $p(e r r)=.14$ and for $b_{2}>c_{1}, p(e r r)=.31$. Note that for the items with high error probabilities, the item specified an ordering not given in the paragraph, but the "greater" of the elements was seen earlier than the element specified as "lesser" in the test item.
Table 2

Mean Times to Read Sentences Presenting Categorical and Ordering Relations

\begin{tabular}{lccccccc}
\hline Condition & $\begin{array}{c}\mathrm{a}> \\
\mathrm{b}_{1}\end{array}$ & $\begin{array}{c}\mathrm{a}> \\
\mathrm{b}_{2}\end{array}$ & $\begin{array}{c}\mathrm{a}> \\
\mathrm{c}_{1}\end{array}$ & $\begin{array}{c}\mathrm{c}_{1}> \\
\mathrm{b}_{1}\end{array}$ & $\begin{array}{c}\mathrm{c}_{2}> \\
\mathrm{b}_{1}\end{array}$ & $\begin{array}{c}\mathrm{c}_{3}> \\
\mathrm{b}_{2}\end{array}$ & $\begin{array}{c}\mathrm{c}_{4}> \\
\mathrm{b}_{2}\end{array}$ \\
\hline $\begin{array}{l}\text { Categorical } \\
\text { Top Down }\end{array}$ & 6.7 & 5.1 & 6.3 & 6.2 & 4.7 & 7.7 & 6.1 \\
$\begin{array}{l}\text { Categorical } \\
\text { Bottom Up }\end{array}$ & 6.2 & 4.7 & 7.2 & 6.5 & 4.0 & 8.5 & 7.1 \\
$\begin{array}{l}\text { Ordering } \\
\text { Top Down }\end{array}$ & 7.5 & 6.2 & 5.9 & 7.3 & 8.6 & 8.1 & 7.7 \\
$\begin{array}{l}\text { Ordering } \\
\text { Bottom Up }\end{array}$ & 6.8 & 9.3 & 6.6 & 8.6 & 6.8 & 8.1 & 7.3 \\
\hline
\end{tabular}

\section{DISCUSSION}

When the relations in one part of a paragraph led to expectations about the semantic content of later sentences, those later sentences were read more quickly than they were when the preceding sentences had not yet been presented. And when a sentence contradicted expectations based on earlier sentences, reading of that sentence and a following sentence was slower. By contrast, weak and insignificant effects of preceding materials on reading time were obtained when relations among sentences did not involve semantic implication.

It should not be suggested that formal structural relations among sentences have no effects of ease of assimilating the information in those sentences. In the categorical paragraphs, a trend was observed in the direction of greater ease for adding nodes to a network than for starting the network, though it was not significant. And the nonsignificant trend toward greater difficulty in adding relations in the networks of orderings might be due to the fact that the relations given did not form a simple linear ordering. Huttenlocher (1968) and Potts (1972) have obtained strong evidence that information about orderings can be stored in a linear array in memory. If Ss have a bias toward using such a linearizing mechanism, this would produce some confusion and interference in the present materials, which are not linearly ordered. The high proportions of errors made on questions about the orderings tend to support the idea that these paragraphs were confusing to the Ss.

The main finding, though, involves the materials where expectations about the semantic content of later sentences would be developed in earlier sentences. Modern theories of language comprehension such as Bobrow's (1968), Quillian's (1968), Schank's (1972), and Winograd's (1971) use the idea that linguistic inputs are interpreted in relation to general conceptual and factual knowledge. This general point leads to the idea that as $\mathrm{S}$ assimilates information, a structure of relations among concepts will be developed by $S$, representing the semantic content of the sentences read, and considerable evidence for this idea has been presented. At any point 
in the process of building a semantic structure, the information already assimilated can give rise to expectations about the content of later sentences. The present study provides evidence that comprehension is made easier when new information is consistent with such semantically derived expectations and is harder when the new information contradicts such expectations.

\section{REFERENCES}

Bobrow, D. G. Natural language input for a computer problem-solving system. In M. Minsky (Ed.), Semantic information processing. Cambridge, Mass: M.I.T. Press, 1968. Pp. 135-215.

Bransford, J. D., \& Franks, J. J. The abstraction of linguistic ideas. Cognitive Psychology, 1971, 2, 331-350.

Huttenlocher, J. Constructing spatial images: A strategy in reasoning. Psychological Review, 1968, 75, 550-560.

Kintsch, W., \& Keenan, J. Reading rate and retention as a function of the number of propositions in the base structure of sentences. In Studies in mathematical learning theory and psycholinguistics, University of Colorado, April 1972.

Kintsch, W., \& Monk, D. Storage of complex information in memory: Some implications of the speed with which inferences can be made. Journal of Experimental Psychology, $1972,94,25-32$.

Potts, G. R. Information processing strategies used in the coding of linear orderings. Journal of Verbal Learning \& Verbal Behavior, 1972, 11, 727-740.

Quillian, M. R. Semantic memory. In M. Minsky (Ed.), Semantic information processing. Cambridge, Mass: M.I.T. Press, 1968. Pp. 216-270.

Sachs, J. Recognition memory for syntactic and semantic aspects of connected discourse. Perception \& Psychophysics, 1967, 2, 437-442.

Schank, R. C. Conceptual dependency: A theory of natural language understanding. Cognitive Psychology, 1972, 3 , 552631 .

Winograd, T. Understanding natural language. Cognitive Psychology, 1972, 3, 1-19.

(Received for publication April 20, 1973; revision received June 25,1973 .) 